\title{
CUIDADOS DE ENFERMAGEM NA PREVENÇÃO DA PNEUMONIA ASSOCIADA À VENTILAÇÃO MECÂNICA
}

\section{Nursing care in preventing pneumonia associated with mechanical ventilationt}

Atención de enfermería en la prevención de la neumonía associada a la ventilación mecânica

Givanilson da Silva Costa • Enfermeiro pelo Centro Universitário Tabosa de Almeida (ASCES/UNITA)•E-mail: givanilsonsilvacostta@gmail.com Daniela Barbosa de Lima • Enfermeira pelo Centro Universitário Tabosa de Almeida (ASCES/UNITA)・E-mail: daniela.blima23@gmail.com

Rebeca Larissa Nepomuceno Torres • Enfermeira pelo Centro Universitário Tabosa de Almeida (ASCES/UNITA)・ E-mail: rebecatorres4004@gmail.com

Maria Lara Costa Manso • Enfermeira pelo Centro Universitário Tabosa de Almeida (ASCES/UNITA) • E-mail: laramlcm@hotmail.com

Oberto César dos Santos • Enfermeiro pelo Centro Universitário Tabosa de Almeida (ASCES/UNITA)・ E-mail: obertocesar.enf@gmail.com

João Augusto da Silva • Enfermeiro pelo Centro Universitário Tabosa de Almeida (ASCES/UNITA)・ E-mail: 2017206050@app.asces.edu.br

Marcos Matheus Missias da Silva • Enfermeiro pelo Centro Universitário Tabosa de Almeida (ASCES/UNITA)・ E-mail: 2017206031@app.asces.edu.br

Diego Augusto Lopes Oliveira • Doutorando em Enfermagem - PPGENF/UFPE • E-mail: diegoaugusto.enf@gmail.com

Rosa Régia Sousa de Medeiros • Docente do curso de Enfermagem -ASCES/UNITA - E-mail: rosamedeiros@app.asces.edu.br

Lidiane Marinho Silva Barbosa • Docente do curso de Enfermagem ASCES/UNITA • E-mail: lidianebarbosa@asces.edu.br

Autor correspondente:

Givanilson da Silva Costa • E-mail: givanilsonsilvacostta@gmail.com 


\section{RESUMO}

Introdução: A ventilação mecânica invasiva é um recurso terapêutico artificial, que possui a finalidade de equilíbrio da ventilação e/ou oxigenação de pacientes com insuficiência respiratória, entre as complicações desta intervenção, destaca-se a pneumonia. Nesse âmbito, o enfermeiro tem um papel primordial na criação de programas de prevenção das infecções, sobretudo na precaução da pneumonia associada à ventilação mecânica nas unidades de terapia intensiva. Objetivo: Descrever as ações de enfermagem para prevenção da pneumonia associada à ventilação mecânica invasiva na Unidade de Terapia Intensiva. Metodologia: Trata-se de um estudo do tipo revisão integrativa da literatura, para qual, definiu-se como ambiente de pesquisa as bases de dados BDENF, LILACS e MEDLINE. Elencou-se artigos publicados nos idiomas português, inglês e espanhol no período entre 2015 a 2019. Explorou-se os artigos a partir dos parâmetros estabelecidos pelo guia PRISMA, verificação dos títulos, resumos e texto completo, considerando os estudos que estivessem de acordo com o objetivo proposto, sendo selecionados 7 artigos para compor esta revisão. Resultados: Observou-se nos artigos analisados a importância da adesão de ferramentas de ações no processo de cuidado, como os protocolos e bundles (um conjunto de medidas e intervenções) de prevenção da pneumonia associada à ventilação mecânica, para quais compreendem práticas como a verificação da pressão do cuff, elevação da cabeceira, aspiração de secreção, higiene oral, validade dos artigos médicos, lavagens das mãos e a interrupção da infusão de sedativos. Conclusões: Preconiza-se a adesão de medidas preventivas da pneumonia associada à ventilação mecânica nas unidades de terapia intensiva, as quais precisam ser praticadas rigorosamente pelos profissionais de enfermagem, por serem representantes do cuidado. Ressalta-se a necessidade de atualizações e construções de protocolos com medidas que possam prevenir as infecções de trato respiratório oriundas do uso da ventilação mecânica invasiva.

Palavras-Chave: Cuidados de Enfermagem; Pneumonia Associada à Ventilação Mecânica; Profissionais de Enfermagem; Qualidade da Assistência à Saúde; Unidades de Terapia Intensiva.

\section{ABSTRACT}

Introduction: Invasive mechanical ventilation is an artificial therapeutic resource, which aims to balance ventilation and / or oxygenation of patients with respiratory failure, among the complications of this intervention, pneumonia stands out. In this context, the nurse has a primary role in creating infection prevention programs, especially in the prevention of pneumonia associated with mechanical ventilation in intensive care units. Objective: To describe nursing actions to prevent pneumonia associated with invasive mechanical ventilation in the Intensive Care Unit. lethodology: This is a bibliographic study, of the type integrative literature review, which the databases BDENF, LILACS and MEDLINE were defined as a research vironment. Articles published in Portuguese, English and Spanish from 2015 to 2019 re listed. Articles were explored using the parameters established by the Preferred porting Items for Systematic Reviews and Meta-Analyzes (PRISMA) guide, fication of titles, abstracts and full text, considering studies that were in accordance the proposed objective, with 7 articles selected to compose this review. Results:It 


\section{ciênncia
pural}

was observed in the analyzed articles the importance of adhering to action tools in the care process, such as protocols and bundles (a set of measures and interventions) for preventing pneumonia associated with mechanical ventilation, for which they comprise practices such as checking the cuff pressure, elevating the headboard, secretion aspiration, oral hygiene, validity of medical articles, hand washing and stopping the infusion of sedatives. Conclusions: Adherence to preventive measures for pneumonia associated with mechanical ventilation in intensive care units is recommended, which must be strictly practiced by nursing professionals, as they are representatives of care. The need to update and build protocols with measures that can prevent respiratory tract infections arising from the use of invasive mechanical ventilation is emphasized.

Keywords: Nursing Care; Pneumonia, Ventilator-Associated; Nurse Practitioners; Quality of Health Care; Intensive Care Units.

\section{RESUMEN}

Introducción: La ventilación mecánica invasiva es un recurso terapéutico artificial, con la finalidad de equilibrar la ventilación y / u oxigenación en pacientes con insuficiencia respiratoria, entre las complicaciones de esta intervención se destaca la neumonía. En este contexto, la enfermera es fundamental en la creación de programas de prevención de infecciones, especialmente en neumonía asociada a ventilador en unidades de cuidados intensivos. Objetivo: Describir las acciones de enfermería para prevenir la neumonía asociada a la ventilación mecánica invasiva en la Unidad de Cuidados Intensivos. Metodología: Se trata de una revisión integradora de la literatura, realizada a través de las bases de datos BDENF, LILACS y MEDLINE. Se enumeraron los artículos publicados en portugués, inglés y español en el período comprendido entre 2015 y 2019. Los artículos fueron explorados en base a los parámetros establecidos por la guía Preferred Reporting Items for Systematic Reviews and Meta-Analyzes (PRISMA), verificación de títulos, resúmenes y texto completo., considerando los estudios que estaban de acuerdo con el objetivo propuesto, seleccionándose 7 artículos. Resultados: Se observó la importancia de la adherencia a herramientas de acción en el proceso de atención, como protocolos y paquetes (conjunto de medidas e intervenciones) para la prevención de la neumonía asociada al ventilador, que incluyen prácticas como el control de la presión del paciente. manguito, elevación de la cabeza, aspiración de secreciones, higiene bucal, vigencia de artículos médicos, lavado de manos e interrupción de la infusión de sedantes. Conclusiones: Se recomienda la adherencia a las medidas preventivas, las cuales deben ser practicadas con rigor por los profesionales de enfermería, por ser representantes del cuidado. Se destaca la necesidad de actualización y construcción de protocolos con medidas que puedan prevenir las infecciones del tracto respiratorio derivadas del uso de ventilación mecánica invasiva.

labras clave: Atención de Enfermería; Neumonía Asociada al Ventilador; fermeras Practicantes; Calidad de la Atención de Salud; Unidades de Cuidados tensivos. 


\section{Introdução}

O termo Infecções Hospitalares $(\mathrm{IH})$ vem sendo substituído nos últimos anos por Infecções Relacionadas à Assistência à Saúde (IRAS), as quais são definidas por seu desenvolvimento no ambiente hospitalar, estando associadas à internação ou quando manifestada até $72 \mathrm{~h}$ após a alta ${ }^{1,2}$. As Unidades de Terapia Intensiva (UTI) são tidas como epicentro da emergência e disseminação de patógenos resistentes, visto que os pacientes admitidos nesta unidade estão sujeitos à alta demanda de procedimentos invasivos, os quais aumentam as chances de desenvolver comorbidades capazes de prolongar o tempo de internação, custos mais elevados e altas taxas de mortalidade ${ }^{3,4}$.

A Ventilação Mecânica Invasiva (VMI) é um recurso terapêutico artificial, que possui finalidade de equilíbrio da ventilação e/ou oxigenação de pacientes que desenvolvem insuficiência respiratória. Dentre as IRAS, destaca-se a Pneumonia Associada à Ventilação Mecânica (PAVM) invasiva, definida como uma infecção pulmonar que pode surgir entre 48 a 72 horas após a intubação endotraqueal, bem como, até 48 horas após a extubação ${ }^{5,1}$. A PAVM é caracterizada por alterações nas radiografias do tórax com presença de um novo ou progressivo infiltrado, sinais de infecção, mudança nas características da expectoração (escarro) e piora dos parâmetros ventilatórios 6,7 .

A PAVM é a segunda infecção nosocomial mais frequente na população crítica e nos pacientes em $\mathrm{VMI}^{8}$. Os fatores de risco para o seu desenvolvimento são classificados em não modificáveis: idade, gravidade do estado de saúde do paciente, assim como, presença de comorbidades (doenças respiratórias, coronarianas), e os modificáveis: rebaixamento do nível de consciência, uso prévio de antimicrobiano, suporte nutricional enteral, broncoaspiração, ambiente (microbiota) da UTI e medidas de assistência inadequada por parte dos profissionais da equipe multidisciplinar ${ }^{2,3}$.

Com a finalidade de contribuir para a redução das infecções causadas por VMI, recomenda-se a utilização de estratégias que englobam o grupo multiprofissional, través da implementação de protocolos direcionados ao uso racional de sedação e \$mame, bem como, medidas eficazes e preventivas na prestação de assistência que vem ser realizadas nas UTIs ${ }^{8,4}$. Nesse contexto, de modo a reduzir a incidência de $\mathrm{M}$, tem sido bastante utilizado o bundle de prevenção (um conjunto de medidas 
e intervenções), que é constituído de práticas baseadas em evidências que quando implementados em conjunto, resultam em reduções significativas das contaminações e agravamentos 3,9 .

A busca pela definição de qualidade em saúde e segurança do paciente, é uma discussão que vem ganhando muito espaço nos últimos anos. $\mathrm{O}$ foco da equipe de enfermagem no cuidado ativo em terapia intensiva, visa atender as necessidades biopsicossociais e espirituais do cliente e familiares ${ }^{10}$. Nesse âmbito, o enfermeiro tem um papel primordial na criação de programas de prevenção das IRAS, sobretudo na precaução de PAVM em ambiente de UTI, devendo intervir principalmente nos fatores modificáveis e na qualidade da assistência ${ }^{2,4}$. Observa-se que em instituições que apresentam protocolos definidos para prevenção de PAV, existe uma alta adesão da equipe de enfermagem, em média $70,16 \%$ realizam as medidas preconizadas, demonstrando a melhoria desse indicador na UTI ${ }^{11}$.

Nessa perspectiva, com intenção de realizar ações efetivas acerca da implantação das medidas de prevenção para PAVM, torna-se necessário que os profissionais realizem uma prática qualificada, oferecendo uma melhor qualidade na assistência ao paciente em VMI, por meio da aplicação consciente dos cuidados e intervenções. Partindo desta premissa, este estudo tem por objetivo descrever as ações de enfermagem para prevenção da pneumonia associada à ventilação mecânica invasiva na Unidade de Terapia Intensiva.

\section{Metodologia}

Trata-se de uma revisão integrativa da literatura, que consiste em um método extensivo de revisão, o qual permite a inserção de estudos empíricos e literários com variadas metodologias, as experimentais e não-experimentais, para o melhor entendimento acerca do fenômeno estudado ${ }^{12,13}$.

A seleção das publicações ocorreu de acordo com Preferred Reporting Items for Systematic Reviews and Meta-Analyses (PRISMA) ${ }^{14}$, apresentado na figura 01, que mbora seja usado para revisões sistemáticas, foi utilizado para maior rigor todológico e os tópicos que não se relacionam com a revisão integrativa foram 
desconsiderados. Estruturou-se o estudo a partir das 6 etapas seguintes: 1- definição do tema e questão norteadora da pesquisa; 2- escolha de critérios de inclusão e exclusão empregados para a seleção na busca de literaturas; 3- realizar a análise dos dados; 4- a avaliação dos estudos incluídos; 5- filtragem e interpretação dos resultados dos artigos selecionados; 6- apresentação do conhecimento oriundos da revisão integrativa e a conclusão, com intuito de alcançar o objetivo proposto ${ }^{12}$.

Utilizou-se a seguinte pergunta para nortear a pesquisa: “Quais as ações de enfermagem desenvolvidas na prevenção da pneumonia associada à ventilação mecânica na unidade de terapia intensiva?".

Empregou-se os descritores provenientes do Descritores em Ciências da Saúde (DeCS) e combinados com o operador booleano "AND", os quais foram consultados separadamente em cada idioma, respectivamente: Cuidados de Enfermagem AND Unidades de Terapia Intensiva AND Pneumonia Associada à Ventilação Mecânica; Atención de Enfermería AND Unidades de Cuidados Intensivos AND Neumonía Asociada al Ventilador; Nursing Care AND Intensive Care Units AND Pneumonia, Ventilator-Associated.

Definiu-se como ambiente para pesquisa, as bases de dados: Bases de Dados Específica da Enfermagem (BDENF), Literatura Latino-Americana e do Caribe em Ciências de Saúde (LILACS) e Medical Literature Analysis and Retrieval System Online (MEDLINE). Nesta etapa do estudo, procurou-se reduzir os prováveis vieses de pesquisa na seleção das publicações encontradas.

Aplicou-se como critérios de inclusão: artigos publicados nos idiomas português, inglês e espanhol, disponíveis em texto completo no período entre 2015 a 2019, por serem sobretudo evidências científicas recentes e atualizadas que portam alta relevância para compor a pesquisa. Empregou-se como critérios de exclusão: publicações que não possuíram adequação à temática do estudo, artigos de revisão integrativa e relato de experiência, resumos e cartas de opinião. Ressalta-se que a esquisa nas bases de dados, foi realizada por meio da avaliação por pares, o qual ta-se de um método que coloca os autores em posição de comparação para refletir ais os achados serão selecionados, desempenhada por dois revisores de forma ividual, para que ocorra uma consonância dos resultados ${ }^{15}$. 
Analisou-se os dados seguindo uma ordem de verificação dos estudos: I- leitura dos títulos, sendo selecionados aqueles que possuíam relação com o tema e objetivo; II- leitura crítica e minuciosa dos resumos, considerando critérios de inclusão, e para último, III- leitura do texto na íntegra, tornando-se apto a compor a pesquisa, aqueles que responderam à pergunta condutora.

Empregando-se o método de Práticas Baseadas em Evidências (PBE) acerca do conhecimento científico na área de enfermagem, seguiu-se a categorização ordenada dos níveis de evidências para aferição da qualidade dos estudos selecionados: Nível Irevisão sistemática ou metanálise; Nível II- estudos controlados e aleatórios; Nível IIIestudos controlados sem randomização; Nível IV- estudos caso-controle ou de coorte; Nível V- revisão sistemática de estudos qualitativos ou descritivos; Nível VI- estudos qualitativos ou descritivos e Nível VII- opiniões ou consensos ${ }^{16}$.

Realizou-se após a etapa de catalogação, a leitura crítica das publicações e posteriormente um produto de análise crítica e interpretação da leitura para o estudo. Apresentou-se o resumo dos resultados em figuras. Subsequente, discutiu-se criticamente os resultados acerca de estudos científicos, sendo elencados conteúdos relevantes e que possuíam adequação à temática da pesquisa.

\section{Resultados}

A busca dos estudos foi realizada no dia 04 de julho de 2020. Encontrou-se 397 estudos com os descritores da pesquisa, respectivamente no idioma português, espanhol e inglês. Por meio da aplicação dos filtros (texto completo, bases de dados, idioma correspondente ao descritor e período dos anos das publicações), resultou em 74 artigos, dos quais passaram pelos critérios de elegibilidade totais. Pela leitura do título selecionou-se 44 publicações, com a análise criteriosa dos resumos, permaneceram 12, dos quais, 07 artigos foram elencados para compor o estudo através da leitura na íntegra. Sendo que, 02 são pertencentes à base de dados BDENF, 03 ao ILACS e 02 a MEDLINE, dos quais, 04 pertencem ao idioma português e 03 ao idioma glês. O processamento dos resultados apresenta-se detalhado na figura 1.

Destacaram-se, em relação ao tipo de delineamento de pesquisa dos estudos liados: um estudo transversal com abordagem quantiqualitativa; um ensaio clínico 
controlado randomizado; uma metanálise de estudos randomizados; um estudo descritivo prospectivo; um estudo transversal com abordagem quantitativa; um estudo observacional longitudinal; um estudo descritivo exploratório com abordagem qualitativa. Entre os estudos utilizados destacam-se dois, dos quais são classificados com níveis I e II, que são considerados como alto nível de evidência, bem como, os demais artigos possuem classificação satisfatória. No ponto de vista das produções, as classificações subsidiam o enfermeiro na avaliação crítica dos resultados de estudos, que servirão para o desenvolvimento do raciocínio clínico, e posteriormente na tomada de decisões, desta forma fundamenta a sua prática em evidências consistentes.
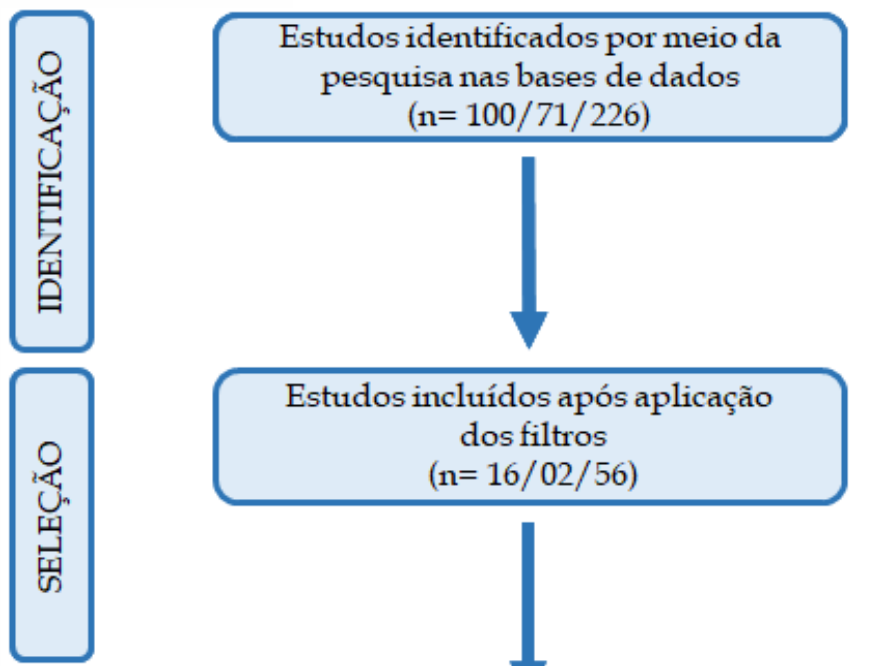

Estudos incluídos após aplicação dos filtros $(\mathrm{n}=16 / 02 / 56)$
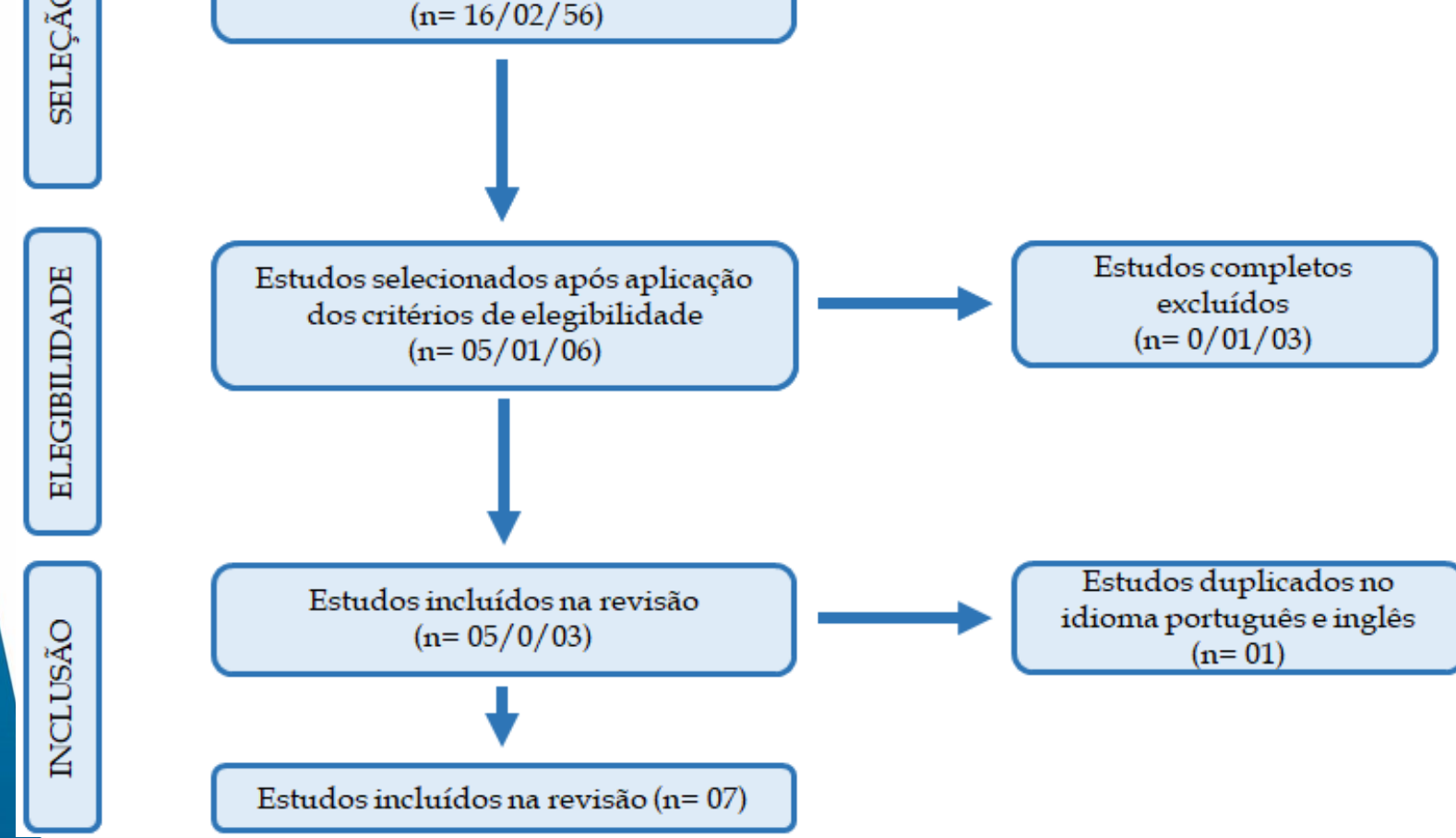

Estudos duplicados no idioma português e inglês $(\mathrm{n}=01)$

Figura 1. Fluxograma com base no protocolo PRISMA correspondente à seleção dos estudos. Caruaru-PE, Brasil, 2020.

Identifica-se no quadro 1, a descrição geral dos artigos selecionados para isão, representados pelo ID, primeiro autor, título da publicação, objetivo, ano e 
periódico do artigo. No quadro 2, apresenta-se as informações dos artigos, detalhadas respectivamente pelos descritores, modalidade da pesquisa, seu nível de evidência e a síntese dos resultados.

Quadro 1. Identificação dos artigos selecionados para compor a revisão. Caruaru-PE, Brasil, 2020.

\begin{tabular}{|c|c|c|c|c|c|}
\hline ID & $\begin{array}{c}\text { Primeiro } \\
\text { autor }\end{array}$ & Ano & Título & Objetivo & Periódico \\
\hline 01 & Melo MM & 2019 & $\begin{array}{l}\text { Pneumonia } \\
\text { Associada à } \\
\text { Ventilação Mecânica: } \\
\text { Conhecimento dos } \\
\text { Profissionais de } \\
\text { Saúde Acerca da } \\
\text { Prevenção e Medidas } \\
\text { Educativas. }\end{array}$ & $\begin{array}{l}\text { Avaliar o conhecimento } \\
\text { dos profissionais de } \\
\text { saúde sobre a prevenção } \\
\text { da PAVM em pacientes } \\
\text { críticos internados nas } \\
\text { Unidades de Terapia } \\
\text { Intensiva e promover } \\
\text { educação permanente } \\
\text { para profissionais das } \\
\text { UTIs sobre prevenção } \\
\text { da PAVM. }\end{array}$ & $\begin{array}{l}\text { Revista Online de } \\
\text { Pesquisa Cuidado é } \\
\text { Fundamental }\end{array}$ \\
\hline 02 & Nobahar M & 2016 & $\begin{array}{lr}\text { Efeitos } & \text { do } \\
\text { enxaguatório bucal } \\
\text { com peróxido de } \\
\text { hidrogênio } & \text { na } \\
\text { prevenção } & \text { de } \\
\text { pneumonia associada } \\
\text { à } \quad \text { ventilação } \\
\text { mecânica r em } \\
\text { pacientes internados } \\
\text { em unidade de } \\
\text { terapia intensiva. }\end{array}$ & $\begin{array}{l}\text { Determinar o efeito do } \\
\text { enxaguatório bucal com } \\
\text { peróxido de hidrogênio } \\
\text { (HP) na incidência de } \\
\text { pneumonia associada à } \\
\text { ventilação mecânica } \\
\text { (PAV) em pacientes } \\
\text { internados na unidade } \\
\text { de terapia intensiva } \\
\text { (UTI). }\end{array}$ & $\begin{array}{l}\text { Revista Brasileira de } \\
\text { Doenças Infecciosas }\end{array}$ \\
\hline 03 & Wen Z & 2017 & $\begin{array}{lr}\text { Drenagem } & \text { de } \\
\text { secreção subglótica } \\
\text { intermitente } & \text { versus } \\
\text { intermitente } & \text { para } \\
\text { prevenir pneumonia } \\
\text { associada } & \text { a } \\
\text { ventilação } & \text { mecânica: } \\
\text { uma } & \text { revisão } \\
\text { sistemática. } & \end{array}$ & $\begin{array}{l}\text { Revisar as evidências } \\
\text { atuais por usar } \\
\text { Drenagem de Secreções } \\
\text { Subglóticas contínua } \\
\text { (CSSD) e Drenagem de } \\
\text { Secreções Subglóticas } \\
\text { intermitente (ISSD), } \\
\text { para comparar as taxas } \\
\text { de complicações de } \\
\text { CSSD e ISSD e para } \\
\text { analisar a escolha entre } \\
\text { CSSD e ISSD. }\end{array}$ & Critical Care Nurse \\
\hline 04 & Barros FRB & 2019 & $\begin{array}{l}\text { Adesão ao bundle de } \\
\text { prevenção de } \\
\text { pneumonia associada } \\
\text { à } \\
\text { mecânicar ventilação }\end{array}$ & $\begin{array}{l}\text { Avaliar a adesão e } \\
\text { conformidade das } \\
\text { práticas que integram } \\
\text { um bundle de } \\
\text { prevenção da PAV em } \\
\text { uma UTI de adultos, } \\
\text { bem como analisar o } \\
\text { impacto dessas medidas } \\
\text { nas taxas de PAV. }\end{array}$ & Revista Cuidarte \\
\hline
\end{tabular}




\begin{tabular}{|c|c|c|c|c|c|}
\hline 05 & Zigart JAA & 2019 & $\begin{array}{l}\text { Adesão ao protocolo } \\
\text { de pneumonia } \\
\text { associado à } \\
\text { ventilação mecânica. }\end{array}$ & $\begin{array}{l}\text { Conhecer a adesão da } \\
\text { equipe de Enfermagem } \\
\text { ao protocolo de } \\
\text { pneumonia associado à } \\
\text { ventilação mecânica nas } \\
\text { Unidades de Terapia } \\
\text { Intensiva. }\end{array}$ & $\begin{array}{lr}\text { Revista } & \text { de } \\
\text { Enfermagem } & \text { UFPE } \\
\text { on line } & \end{array}$ \\
\hline 06 & $\begin{array}{l}\text { Lourençone } \\
\text { SEM }\end{array}$ & 2019 & $\begin{array}{lr}\text { Adesão às } & \text { medidas } \\
\text { preventivas } & \text { versus } \\
\text { incidência } & \text { de } \\
\text { pneumonia associada } \\
\text { à } & \text { ventilação } \\
\text { mecânica. } & \end{array}$ & $\begin{array}{l}\text { Avaliar a taxa de adesão } \\
\text { das ações preventivas } \\
\text { da equipe de } \\
\text { enfermagem para PAV } \\
\text { após a reestruturação e } \\
\text { aplicação do protocolo } \\
\text { de prevenção e verificar } \\
\text { as taxas de densidade } \\
\text { de incidência de } \\
\text { pacientes com PAV. }\end{array}$ & $\begin{array}{lr}\text { Revista } & \text { de } \\
\text { Epidemiologia } & \mathrm{e} \\
\text { Controle } & \mathrm{de} \\
\text { Infecção } & \end{array}$ \\
\hline 07 & Dutra LA & 2019 & $\begin{array}{l}\text { Pneumonia associada } \\
\text { àr ventilação } \\
\text { mecânica: percepção } \\
\text { dos profissionais de } \\
\text { enfermagem. }\end{array}$ & $\begin{array}{l}\text { Apreender a percepção } \\
\text { dos profissionais de } \\
\text { Enfermagem sobre a } \\
\text { segurança do paciente } \\
\text { em ventilação mecânica } \\
\text { com vistas à prevenção } \\
\text { da PAV. }\end{array}$ & $\begin{array}{lr}\text { Revista } & \text { de } \\
\text { Enfermagem } & \text { UFPE } \\
\text { on line } & \end{array}$ \\
\hline
\end{tabular}

Quadro 2. Síntese dos estudos acerca da prevenção da Pneumonia Associada à Ventilação Mecânica (PAVM). Caruaru-PE, Brasil, 2020.

\begin{tabular}{|c|c|c|c|c|}
\hline ID & Descritores & $\begin{array}{l}\text { Modalidade de } \\
\text { pesquisa }\end{array}$ & $\begin{array}{l}\text { Nível de } \\
\text { evidência }\end{array}$ & Síntese dos resultados \\
\hline 01 & $\begin{array}{lr}\text { Pneumonia } & \text { Associada à } \\
\text { Ventilação } & \text { Mecânica, } \\
\text { Unidades de } & \text { Terapia } \\
\text { Intensiva, } & \text { Infecção } \\
\text { Hospitalar, } & \text { Educação } \\
\text { Permanente. } & \end{array}$ & $\begin{array}{l}\text { Estudo } \\
\text { transversal, } \\
\text { quanti-qualitativo. }\end{array}$ & VI & $\begin{array}{l}\text { Identifica-se a recomendação } \\
\text { para lavagem das mãos } \\
\text { utilizando sabonetes líquidos } \\
\text { com antissépticos como a } \\
\text { clorexidina, ressalta-se a } \\
\text { estimulação para o uso de } \\
\text { solução alcoólica. A cabeceira } \\
\text { deve estar elevada em } 15 \text { a } 30 \\
\text { graus para neonatos e } 30 \text { a } 45 \\
\text { graus a partir da fase lactente. A } \\
\text { pressão do cuff deve } \\
\text { permanecer entre } 20 \text { e } 25 \\
\text { mmHg, com checagem a cada } 8 \\
\text { horas. A higiene bucal deve ser } \\
\text { realizada com clorexidina. } \\
\text { Deve-se monitorar o nível de } \\
\text { sedação através de escalas ou } \\
\text { monitores de atividade cerebral } \\
\text { e realizar a interrupção diária de } \\
\text { sedação, quando o quadro } \\
\text { clínico permitir. Os pesquisados } \\
\text { também ressaltaram a } \\
\text { prevenção a partir dos cuidados } \\
\text { e técnicas de aspiração do tubo }\end{array}$ \\
\hline
\end{tabular}




\section{ciência plural}

\begin{tabular}{|c|c|c|c|c|}
\hline & & & & $\begin{array}{lcc}\text { orotraqueal } & \text { e } & \text { traqueostomia, } \\
\text { parâmetros } & \text { ventilatórios e } \\
\text { controle } & \text { de } & \text { etiologias } \\
\text { associadas à PAVM. } & \end{array}$ \\
\hline 02 & 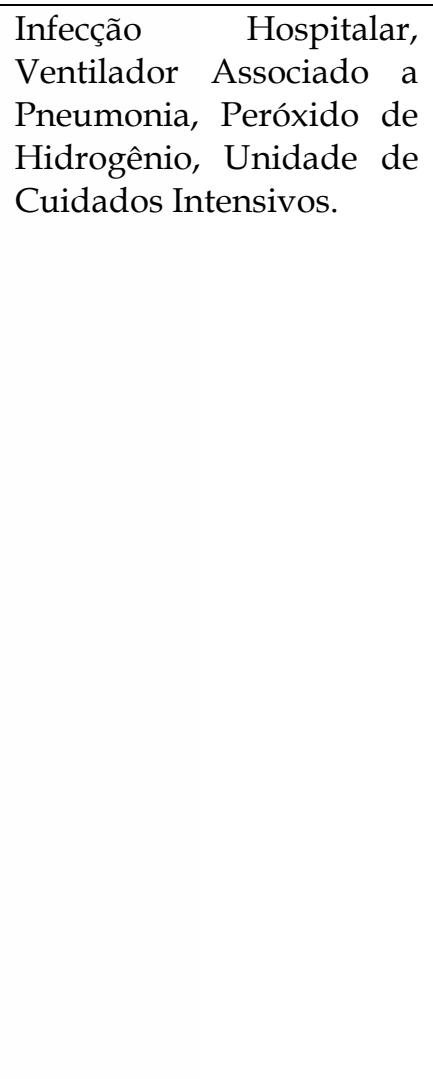 & $\begin{array}{l}\text { Ensaio clínico } \\
\text { controlado } \\
\text { randomizado. }\end{array}$ & II & $\begin{array}{l}\text { O estudo mostra que a } \\
\text { utilização do enxaguante bucal } \\
\text { contendo Peróxido de } \\
\text { Hidrogênio (PH) foi associado a } \\
\text { outras medidas para prevenção } \\
\text { da PAV, como elevação da } \\
\text { cabeceira, pressão do balonete e } \\
\text { medidas gerais de prevenção de } \\
\text { infecções. Iniciou-se o uso nas } \\
\text { primeiras } 24 \text { de internação, } \\
\text { com o uso de luvas estéreis, a } \\
\text { mucosa oral, a língua e a } \\
\text { gengiva do paciente foram } \\
\text { lavadas com } 4-6 \text { cotonetes } \\
\text { embebidos em } 15 \text { cc de PH a } 3 \% \text {, } \\
\text { repetidos } 2 \text { vezes ao dia. O } \\
\text { enxaguante bucal com PH } \\
\text { analisado no presente estudo } \\
\text { tem um efeito bactericida em } \\
\text { bactérias anaeróbicas orais e, ao } \\
\text { desinfetar a cavidade oral, evita } \\
\text { a aspiração de secreções } \\
\text { contaminadas com bactérias no } \\
\text { trato pulmonar inferior, } \\
\text { reduzindo assim a PAV. }\end{array}$ \\
\hline 03 & $\begin{array}{lr}\text { Sucção } & \\
\text { Subglótica, } & \text { Drenagem } \\
\text { Subglótica, } & \text { Secreção } \\
\text { Subglótica, } & \text { Pneumonia } \\
\text { Associada à Ventilação } \\
\text { Mecânica, VAP, Vias } \\
\text { Aéreas } & \text { Artificiais, } \\
\text { ventilação, Intubação. }\end{array}$ & $\begin{array}{l}\text { Metanálise } \\
\text { abrangente e } \\
\text { Sistemática de } \\
\text { estudos } \\
\text { randomizados. }\end{array}$ & I & $\begin{array}{l}\text { O estudo caracteriza a } \\
\text { Drenagem de Secreção } \\
\text { Subglótica (SSD) como forma de } \\
\text { prevenção da PAV, } \\
\text { comparando a forma contínua } \\
\text { (CSSD) com a intermitente } \\
\text { (ISSD), não encontraram } \\
\text { resposta sobre alguma ser mais } \\
\text { eficaz que a outra, elas possuem } \\
\text { efeitos semelhantes e positivos. } \\
\text { Contudo, apontam uma } \\
\text { vertente negativa dessa prática, } \\
\text { a ocorrência de lesão na mucosa. }\end{array}$ \\
\hline 04 & $\begin{array}{lcr}\text { Unidades } & \text { de } & \text { Terapia } \\
\text { Intensiva; } & \text { Pneumonia } \\
\text { Associada à Ventilação } \\
\text { Mecânica; Assistência à } \\
\text { Saúde; Enfermagem. }\end{array}$ & $\begin{array}{l}\text { Estudo descritivo } \\
\text { com abordagem } \\
\text { prospectiva. }\end{array}$ & VI & $\begin{array}{l}\text { O estudo apresenta o bundle de } \\
\text { prevenção da PAVM, que } \\
\text { elenca seis práticas: } \\
\text { posicionamento da cabeceira } \\
\text { entre } 30 \text { a } 45 \text { graus, cuidados } \\
\text { com aspirações de secreções, } \\
\text { higiene oral com clorexidina a } \\
0,12 \% \text {, fisioterapia respiratória e } \\
\text { motora, pressão do cuff entre } \\
20-30 \mathrm{cmH} O \text { e interrupção da } \\
\text { infusão de sedativos. Dessas } \\
\text { práticas, evidenciou-se a maior } \\
\text { frequência feita pelos } \\
\text { profissionais de saúde, }\end{array}$ \\
\hline
\end{tabular}




\begin{tabular}{|c|c|c|c|c|}
\hline & & & & $\begin{array}{l}\text { respectivamente, } \\
\text { posicionamento da cabeceira } \\
\text { entre } 30 \text { a } 45 \text { graus, cuidados } \\
\text { com aspirações de secreções e } \\
\text { fisioterapia respiratória e } \\
\text { motora. }\end{array}$ \\
\hline 05 & $\begin{array}{l}\text { Enfermagem, Protocolos, } \\
\text { Pneumonia, Respiração } \\
\text { Artificial, Unidades de } \\
\text { Terapia Intensiva, Ensino. }\end{array}$ & $\begin{array}{l}\text { Estudo } \\
\text { transversal, } \\
\text { descritivo com } \\
\text { abordagem } \\
\text { quantitativa. }\end{array}$ & VI & $\begin{array}{l}\text { Constatou-se que a cabeceira } \\
\text { elevada entre } 35 \text { a } 45 \text { graus foi o } \\
\text { item mais realizado do } \\
\text { protocolo de prevenção da } \\
\text { PAV, seguida de identificação e } \\
\text { validade dos artigos } \\
\text { respiratórios, sendo esses } \\
\text { materiais a extensão da } \\
\text { aspiração, o kit de inalação, o kit } \\
\text { de nebulização e o filtro HME. }\end{array}$ \\
\hline 06 & $\begin{array}{lr}\text { Pneumonia } & \text { Associada à } \\
\text { Ventilação } & \text { Mecânica, } \\
\text { Infecção } & \text { Hospitalar, } \\
\text { Unidades } & \text { de Terapia } \\
\text { Intensiva, } & \text { Cuidados } \\
\text { Críticos. } & \end{array}$ & $\begin{array}{l}\text { Estudo } \\
\text { observacional, } \\
\text { longitudinal. }\end{array}$ & IV & $\begin{array}{l}\text { O estudo apresenta quatro } \\
\text { medidas que são empregadas } \\
\text { para prevenção da PAV, sendo } \\
\text { elas, o correto posicionamento } \\
\text { do filtro, a manutenção da } \\
\text { cabeceira elevada entre } 30 \text { e } 45 \\
\text { graus, a utilização da } \\
\text { clorexidina para higiene oral e a } \\
\text { mensuração da pressão do } \\
\text { balonete. }\end{array}$ \\
\hline 07 & $\begin{array}{lr}\text { Pneumonia; } & \text { Respiração } \\
\text { Artificial; } & \text { Cuidados } \\
\text { Críticos; } & \text { Unidades de } \\
\text { Terapia } & \text { Intensiva; } \\
\text { Enfermagem; } & \text { Segurança } \\
\text { do Paciente. } & \end{array}$ & $\begin{array}{ll}\text { Estudo } & \text { com } \\
\text { abordagem } & \\
\text { qualitativa, } & \\
\text { descritiva } & \text { e } \\
\text { exploratória. } & \end{array}$ & VI & $\begin{array}{l}\text { O estudo demonstra que a } \\
\text { aspiração de secreções das vias } \\
\text { respiratórias em pacientes com } \\
\text { VMI, é uma medida preventiva } \\
\text { importante, não havendo } \\
\text { diferença significante do } \\
\text { sistema de drenagem fechado } \\
\text { para o aberto. Bem como outras } \\
\text { medidas são citadas, a redução } \\
\text { de infecção cruzada, } \\
\text { principalmente por lavagem e } \\
\text { higienização das mãos } \\
\text { adequadas, realização de } \\
\text { higiene oral, cuidados com } \\
\text { equipamentos, movimentação e } \\
\text { posicionamento do paciente, } \\
\text { verificação da pressão do cuff e } \\
\text { o posicionamento da cabeceira } \\
\text { elevada de } 30 \text { a } 45 \text { graus. }\end{array}$ \\
\hline
\end{tabular}

\section{iscussão}

Uma das medidas profiláticas utilizadas é a higienização das mãos, seja com a e sabão líquido, clorexidina $0,12 \%$ ou álcool a 70\%, a qual deve ser executada com r na realização da sua técnica e periodicidade, antes e após a ocorrência do contato 
direto com o paciente, ou indireto com produtos e equipamentos próximos a este ${ }^{1}$. Considerando a UTI como um local onde concentra-se um grande número de bactérias multirresistentes, essas unidades devem possuir lavabos em locais estratégicos, que contenham dispensadores de soluções antissépticas para o uso de toda a equipe multidisciplinar ${ }^{2,17}$.

A execução da lavagem das mãos é considerada uma medida de maior eficácia para prevenção das IRAS, visto que possibilita a diminuição da microbiota da pele, interrompe a cadeia de transmissão de doenças e dificulta a transmissão cruzada de microrganismos 4,18 . Entretanto, estudos apontam a adesão insuficiente dos profissionais de saúde na realização dessa prática, o que repercute negativamente na assistência prestada, contribui para a reinfecção, compromete a segurança dos profissionais e dos pacientes, além de acarretar no risco de desenvolvimento de PAVM $2,8,19$.

A manutenção da cabeceira dos leitos constantemente elevada em $30^{\circ}$ a $45^{\circ}$ é um dos componentes de cuidados para a prevenção da PAVM, sendo uma medida simples e efetiva, que não demanda custos adicionais e pode ser implementada por todos os profissionais que se encontram na assistência direta ao paciente ${ }^{4,11}$. Destacase que o posicionamento correto do paciente assegura a complacência do seu sistema respiratório, promove a diminuição de refluxo e da aspiração de secreções nasofaríngeas, orofaríngeas e gastrointestinal, o que proporciona o maior volume corrente, reduzindo a chance de contaminação das vias aéreas inferiores 9,18.

Outra intervenção que pode ser realizada em pacientes em ventilação mecânica é a monitorização da pressão do cuff do tubo orotraqueal, no qual o profissional deve realizar a checagem a cada 8 horas e seus valores devem permanecer entre 25 e 30 $\mathrm{cmH}_{2} \mathrm{O}$. A fim de evitar o comprometimento da microcirculação da traqueia, não se recomenda valores maiores que os mensurados, uma vez que podem causar lesões na quéia, como perda de cílios, descamação do epitélio, estenose e isquemia devido à essão excessiva. Entretanto, a pressão insuficiente pode favorecer a passagem de reções subglóticas localizadas acima do cuff e ocasionar aspiração1,6,17. 
A utilização do Peróxido de Hidrogênio para higienização oral (PH) mostrou uma importante ação sobre o controle proliferativo do biofilme, associando-se a ação das bactérias orais residentes do gênero Streptococcus, que são produtoras de $\mathrm{PH}$ e atuam no controle de outras bactérias, todavia o uso deve apresentar cautela, por estar ligada a causas de queimaduras quando diluído incorretamente ${ }^{20-22}$. A solução mais utilizada na limpeza bucal é o digluconato de clorexidina $0,12 \%$, a qual é considerada padrão ouro para esta finalidade por se tratar de um antisséptico com amplo espectro, no qual deve ser utilizada pelos profissionais de saúde a cada 12 horas, 90,17 .

A proliferação microbiana torna-se mais abundante diante do excesso de secreção nas estruturas pulmonares de passagens, que em geral, surge decorrente da diminuição da resposta nervosa vagal, que culmina na tosse ${ }^{1}$. Devendo assim ocorrer a aspiração das vias aéreas, no entanto, quando realizada de forma inapropriada implica em fatores contribuintes para o desenvolvimento da PAVM, além de poder proporcionar lesões nas estruturas e não sucção efetiva das secreções. O sistema fechado apresenta vantagens consideráveis, como redução do risco de queda da saturação de oxigênio devido a desconexão da VM, a diminuição da ameaça de exposição e propagação dos patógenos suspensos no ar decorrente da aspiração17,18,23.

Outra estratégia utilizada pelos profissionais da assistência para diminuir a incidência de PAVM consiste na interrupção diária da sedação, visto que altas doses podem resultar em lesões neurológicas, esvaziamento gástrico lento, sendo capaz de ocasionar diarreia e principalmente náuseas, aumentando a predisposição do paciente a broncoaspiração $2,10,17$. Essa medida compõe um conjunto de boas práticas, uma vez que verifica a possibilidade de extubação precoce, que apresenta como finalidade reduzir o tempo de utilização da VM e diminuir os efeitos adversos relacionados a esta assistência. Contudo, é imprescindível a cautela na realização desta conduta, sendo que a extubação precoce executada por equívoco, pode acarretar em reintubação 1,6,24.

Como forma de prevenir a colonização por microrganismos associados a VM, é icado a troca do filtro umidificador Heatand Moisture Exchanger (HME) quando ver condensação, sujidades ou danos, recomenda-se na ausência de quaisquer ses fatores, a troca no período de 5 a 7 dias ${ }^{3,10}$. Preconiza-se que os profissionais de 
saúde estejam atentos ao posicionamento desses filtros, os quais devem estar localizados acima da inserção do tubo e em bom estado de conservação. Nota-se que os estudos encontrados divergem quanto ao seu tempo de validade, alternando entre um a sete dias ou em casos de sujidades, condensação e danos, embora não exista um consenso sobre esse prazo 7,11

Pode-se apontar como limitações do estudo a dificuldade de encontrar artigos com o corte temporal da pesquisa, sobre os benefícios da utilização do enxaguante com PH na prática clínica, visto que não foram encontradas muitas evidências sobre esta medida. Outra limitação está relacionada à validade dos filtros, uma vez que não ocorreu consenso sobre a validade da utilização, o qual configura-se como uma das medidas de prevenção da PAVM, que deve ser verificada durante a assistência aos pacientes que estão sob uso da VMI. Tais implicações podem servir como base para o desenvolvimento de futuras pesquisas clínicas, sejam experimentais ou quase experimentais, pois podem contribuir para a melhora no prognóstico do paciente crítico e otimização dos custos hospitalares.

\section{Conclusões}

Constatou-se diversas medidas preventivas a pneumonia associada à ventilação mecânica, as quais precisam ser praticadas rigorosamente pelos profissionais de enfermagem, uma vez que possuem grande responsabilidade na prevenção de complicações e no cumprimento de protocolos, compostos por: lavagens das mãos, controle da pressão do cuff, elevação da cabeceira, higienização da cavidade oral, aspiração das vias aéreas, validade dos artigos médicos, bem como, busca e incentivo de educação permanente nas UTIs.

A partir do disposto, é sine qua non sempre atualizações e construções de protocolos com medidas que possam prevenir as infecções do trato respiratório riundas do uso da VMI, como também, um maior aprofundamento no que diz peito a acurácia das medidas de prevenção a esses agravos. Assim, espera-se que revisão contribua para o avanço do conhecimento no âmbito das medidas 
profiláticas para a PAV, embasada em parâmetros atuais e fidedignos para elevação da performance assistencial.

\section{Referências}

1. Silva G, Souza V, Lopes D, Oliveira JL, Fernandes L, Tonini N, et al. Práticas De Prevenção De Pneumonia Associada À Ventilação Mecânica Em Terapia Intensiva. Revista Enfermagem Atual In Derme. 2019; 90(28).

2. Nascimento TBP, Ramos CH, Silva TS, Torres VPS. Efetividade das medidas de prevenção e controle de pneumonia associada à ventilação mecânica na UTI. Perspectivas Online: Biológicas e Saúde. 2017; 7(25): 1-24.

3. Lourençone EM, Branco A, Monteiro AB, Fonseca JP, Caregnato RCA. Adesão às medidas preventivas versus incidência de pneumonia associada à ventilação mecânica. Revista de Epidemiologia e Controle de Infecção. 2019; 9(2): 1-7.

4. Leal GA, Ribeiro JB, Santos JJ, Cavalcante AB. Cuidados de enfermagem para prevenção da pneumonia associada à ventilação mecânica em unidades de terapia intensiva: uma revisão literária. Ciências Biológicas e de Saúde Unit. 2017; 4(1): 95-108.

5. Amaral JM, Ivo OP. Prevenção de pneumonia associada à ventilação mecânica. Revista Enfermagem Contemporânea. 2016; 5(1): 109-117.

6. Alecrim RX, Taminato M, Belasco AGS, Barbosa D, Kusahara DM, Fram D. Boas práticas na prevenção de pneumonia associada à ventilação mecânica. Rev Acta paul. enferm. 2019; 32(1).

7. Pires FC, Fanan JMV, Nascimento JSG. Ações de cuidado para a promoção da segurança ao paciente em ventilação mecânica invasiva. Rev Enferm UFSM. 2017; 7(3): 411-423.

8. Romero DMP, Reboredo MM, Gomes EP, Coelho CM, Paula MAS, Souza LC, et al. Efeitos da implementação de um programa de educação de higienização das mãos entre profissionais de uma UTI: análise de séries temporais interrompidas. Jornal Brasileiro de pneumologia. 2019; 45(5).

Barros FRB. Adesão ao bundle de prevenção de pneumonia associada à ventilação mecânica. Rev Cuidarte. 2019; 10(2): e746.

Brabo CFB, Zeitoun SS. Pneumonia associada à ventilação mecânica: avaliação do hecimento da equipe de enfermagem de uma terapia intensiva. Arq Med Hosp Fac enc Med Santa Casa São Paulo. 2017; 62(3):130-8. 
11. Zigart JAA, Contrin LM, Beccaria LM, Frutuoso IS, Silveira AM, Werneck AL. Adesão ao protocolo de pneumonia associado à ventilação mecânica. Rev enferm UFPE on line. 2019; 13(1): 655-63.

12. Souza MT, Silva MD, Carvalho R. Revisão integrativa: o que é e como fazer. Einstein (São Paulo). 2010; 8(1): 102-106.

13. Botelho LLR, Cunha CCA, Macedo, M. O método da revisão integrativa nos estudos organizacionais. Gestão e sociedade. 2011; 5(11): 121-136.

14. Moher D, Liberati A, Tetzlaff J, Altman DG, The PRISMA Group Preferred Reporting Items for Systematic Reviews and Meta-Analyses: The PRISMA Statement. PLOS Medicine. 2009; 6(7): e1000097.

15. Stumpf, I. Avaliação pelos pares nas revistas de comunicação: visão dos editores, autores e avaliadores. Perspectivas em Ciência da Informação. 2008; 13(1): 18-32.

16. Pompeo DA, Rossi LA, Galvão CM. Revisão integrativa: etapa inicial do processo de validação de diagnóstico de enfermagem. Acta paul. enferm. 2009; 22(4): 434-8.

17. Melo MM, Santiago LMM, Nogueira DL, Vasconcelos MFP. Ventilator-Associated Pneumonia: the Knowledge of Health Professionals Towards Prevention and Educational Measures / Pneumonia Associada à Ventilação Mecânica: Conhecimento dos Profissionais de Saúde Acerca da Prevenção e Medidas Educativas. Revista de Pesquisa: Cuidado é Fundamental Online. 2019; 11(2): 377-382.

18. Dutra LA, Esteves LO, Silva TO, Resck ZMR, Lima RS, Sanches RS. Pneumonia associada à ventilação mecânica: percepção dos profissionais de enfermagem. Rev enferm UFPE on line. 2019; 13(4): 884-92.

19. Souza LM, Ramos MF, Becker ESS, Meirelles LCS, Monteiro SAO. Adesão dos profissionais de terapia intensiva aos cinco momentos da higienização das mãos. Rev. Gaúcha Enferm. 2015; 36(4): 21-28.

20. Nobahar M, Razavi MR, Malek F, Ghorbani R. Effects of hydrogen peroxide mouthwash on preventing ventilator-associated pneumonia in patients admitted to the intensive care unit. The Brazilian Journal of Infectious Diseases. 2016; 20(5): 444450.

21. Zhang $\mathrm{K}$, Zhou $\mathrm{X}, \mathrm{Xu} \mathrm{X}$. The origin of hydrogen peroxide in oral cavity and its role in oral microecology balance. West China Journal Of Stomatology. 2017; 35(2): 215-220.

22. Fonseca B, Bocassanta ACS, Bozza A, Fosquiera EC. Microrganismos bucais no desenvolvimento da pneumonia aspirativa por ventilação mecânica em pacientes de unidade de terapia intensiva- revisão de literatura, Revista UNINGÁ. 2017; 30(2): 3743 
23. Wen Z, Zhang H, Ding J, Wang Z, Shen M. Continuous Versus Intermittent Subglottic Secretion Drainage to Prevent Ventilator-Associated Pneumonia: A Systematic Review. Critical Care Nurse. 2017; 37(5) 10-17.

24. Amaral JM, Ivo OP. Prevenção de pneumonia associada à ventilação mecânica. Revista Enfermagem Contemporânea. 2016; 5(1): 109-117. 